\title{
A sexualidade na percepção dos acadêmicos da Faculdade Guaraí - FAG
}

A sexualidade é tida como uma das primícias da humanidade, isso por suas características multifacetárias que podem influenciar em todos os aspectos e áreas da vida. Tem como objetivo em caracterizar o comportamento sexual e sua implicância na vida dos acadêmicos. Este estudo é uma pesquisa de campo de abordagem quantitativa, exploratório-descritivo. A população deste estudo é composta por 146 acadêmicos de todos os cursos da faculdade. Os resultados surgidos na pesquisa são de maioria concordante com autores conhecidos de nível nacional, os mesmos contemplam os seguintes: $O$ comportamento sexual dos acadêmicos entrevistados apontou que todos já possuem uma vida sexualmente ativa. A maioria possui parceiro fixo ( $82 \%)$ e em decorrência deste fator, apenas uma minoria faz uso do preservativo (14\%), por confiarem em seus parceiros (as), relatando que o uso do preservativo é desconfortável (70\%). Tal comportamento acaba expondo os participantes a ocorrência de uma gravidez não planejada ou IST, sendo que as IST's são mais propensas a acontecerem, levando em consideração que de 146 participantes, 62 indivíduos utilizam preservativo e 84 fazem uso de demais métodos contraceptivos, deixando claro que a principal intenção é prevenir uma possível gravidez indesejada. Conclui-se então que os resultados indicam falhas no auto cuidado dos jovens, que adotaram comportamentos errôneos pela ausência de educação sexual.

Palavras-chave: Educação e comportamento sexual; Prevenção de doenças; Práticas sexuais.

\section{Sexuality in the perception of academics at Faculdade Guaraí-FAG}

Sexuality is seen as one of the first fruits of humanity, due to its multifaceted characteristics that can influence in all aspects and areas of life. This study is a field research with a quantitative, exploratory-descriptive approach. The population of this study is composed of 146 academics from all courses at the college. The results of the research are mostly in agreement with well-known authors at national level, they include the following: The sexual behavior of the interviewed academics pointed out that everyone already has a sexually active life. Most have a steady partner (82\%) and as a result of this factor, only a minority use condoms $(14 \%)$, because they trust their partners, reporting that condom use is uncomfortable (70\%). Such behavior ends up exposing participants to the occurrence of an unplanned pregnancy or STI, and STIs are more likely to happen, taking into account that of 146 participants, 62 individuals use condoms and 84 use other contraceptive methods, making it clear that the main intention is to prevent a possible unwanted pregnancy. It is concluded then that the results indicate flaws in the self-care of young people, who adopted erroneous behaviors due to the lack of sexual education.

Keywords: Sexual education and behavior; Prevention of diseases; Sexual practices.

Topic: Enfermagem em Saúde Pública

Reviewed anonymously in the process of blind peer.
Received: 28/03/2021

Approved: 07/04/2021
Jane Aparecida da Silva Dias

Faculdade Guaraí, Brasil

http://lattes.cnpq.br/0037759374568107

janeaparecida@hotmail.com

Letícia Helena Teixeira Marin

Faculdade Guaraí, Brasil

http://lattes.cnpq.br/4254129356605862

leticiahelenamarin@hotmail.com

Adriana Keila Dias (iD)

Faculdade Guaraí, Brasil

http://lattes.cnpq.br/2128882976477548

http://orcid.org/0000-0003-1291-5593

adrianakeiladias@hotmail.com
Giullia Bianca Ferraciolli do Couto (D)

Faculdade Guaraí, Brasil

http://lattes.cnpq.br/0109560699727614

http://orcid.org/0000-0002-9768-778X

giulliabianca@hotmail.com

Juliane Marcelino dos Santos (iD

Faculdade Guaraí, Brasil

http://lattes.cnpq.br/8751832120632358

http://orcid.org/0000-0002-2059-1069

julianemarcelino@hotmail.com

Reobbe Aguiar Pereira (ic

Faculdade Guaraí, Brasil

http://lattes.cnpq.br/7447115724350334

http://orcid.org/0000-0003-2578-2611

enfreobbe@gmail.com
Glaucya Wanderley Santos Markus (ic)

Faculdade Guaraí, Brasil

http://lattes.cnpq.br/5568510365985231

http://orcid.org/0000-0001-8916-1086

glaucyamarkus@outlook.com

\section{Referencing this:}

DIAS, J. A. S.; MARIN, L. H. T.; DIAS, A. K.; SANTOS, J. M.; COUTO, G. B. F.; PEREIRA, R. A.; MARKUS, G. W. S.. A sexualidade na percepção dos acadêmicos da Faculdade Guaraí - FAG. Scire Salutis, v.11, n.2, p.109118, 2021. DOI: http://doi.org/10.6008/CBPC2236-

9600.2021.002.0012 


\section{INTRODUÇÃO}

A sexualidade é tida como uma das primícias da humanidade, isso por suas características multifacetárias que podem influenciar em todos os aspectos e áreas da vida. Desta forma, a sexualidade não se resume apenas ao ato sexual mesmo esse sendo a consolidação, ela está relacionada com os conceitos de saúde e bem estar além do entendimento e aplicação de prevenção e doença, evidenciando sua pluralidade (CARNEIRO et al., 2015).

No entanto, com o passar do tempo, a vasta e desprendida argumentação sobre sexualidade acabou sendo afetada por inúmeros motivos, resultando em perspectivas distorcidas, tabus sexuais, mitos, crendices e subjetividades falsas (FIGUEIROA et al., 2017).

O comportamento sexual e seu entendimento tem ganhado grande espaço dentro do contexto de pesquisas e iniciações cientificas. Isso por que cresce de forma incisiva a disseminação de IST's e também da fecundação não desejada ou não planejada, dessa forma é de suma importância e de interesse público a discussão sobre a temática, pois dessa maneira surgem novas perspectivas para o enfrentamento dessa situação (NASCIMENTO et al., 2018).

O entendimento sobre sexualidade e as práticas sexuais têm suas particularidades, isso acontece devido a todo o seguimento de conceituação que nasce a partir de um contexto cultural e comprovadamente socioeconômico, com isso a caracterização desse público é uma estratégia de enfrentamento. Essa problemática se incorpora em todos os setores da sociedade, e fazem com que a saúde pública e a opinião científica se movimentem para o debate. O ciclo sexual é uma ocasião que abrange diversas características, pois, não compreende apenas questões físicas, mas interfere em todos os quesitos pessoais, que vai desde questões psicológicas até sociais (KERNTOPF et al., 2018).

Os problemas sexuais podem acarretar problemas crônicos e que podem se arrastar por toda a vida dos acadêmicos, ficando evidente a importância de se abordar esse assunto. Além da cronicidade de muitos problemas que a irresponsabilidade ou a má conduta sexual podem gerar, existe ainda a influência que acontece de maneira direta nos estudos e na continuidade dos cursos desses acadêmicos, pois, em muitos casos principalmente em gravidez não planejada a demanda de tempo e fatores clínicos interferem na programação acadêmica, levando ao constrangimento e frustação (OLLIVEIRA et al., 2016).

A partir dessa análise e entendimento do contexto das práticas sexuais. Surgem algumas hipóteses e indagações: De que forma o comportamento sexual interfere na vida dos acadêmicos? Essas implicâncias acontecem de forma positiva ou negativa? Quais as consequências que a sexualidade desregrada tem trazido para esses estudantes?

Diante disso, este artigo tem como objetivo geral caracterizar o comportamento sexual e sua implicância na vida dos acadêmicos, fazendo-se compreender como objetivos específicos à percepção dos acadêmicos do Instituto Educacional Santa Catarina sobre o conceito de sexualidade; identificando o comportamento e aspectos sexuais e fatores de risco associados ao exercício da sexualidade. 


\section{METODOLOGIA}

Este estudo é uma pesquisa de campo de abordagem quantitativa, exploratório e descritivo. 0 prosseguimento do estudo atendeu às normas nacionais e internacionais de ética em pesquisa envolvendo seres humanos, resguardados pela a Resolução no 466, de 12 de outubro de 2012 do Conselho Nacional de Saúde (CNS). A pesquisa obteve a aprovação do Comitê de Ética em Pesquisa da Universidade Estadual do Tocantins - UNITINS, campus de Palmas-TO, emitido sob o Parecer no 4.329.972.

A população deste estudo é composta por acadêmicos de todos os cursos da faculdade (Enfermagem, Direito, Educação Física, Administração, Ciências Biológicas, Biomedicina, Agronomia, Engenharia Civil, Fisioterapia, Pedagogia e Zootecnia). Os critérios de inclusão da pesquisa foram todos os acadêmicos acima de 18 anos matriculados na instituição, que responderem já ter tido vida sexual ativa e que tenham respondido o questionário por completo. Nesta pesquisa, a seleção dos sujeitos não será intencional, de acordo com os critérios de inclusão a mesma será realizada entre os acadêmicos aleatórios de ambos os sexos e em diferentes cursos de graduação da faculdade, (Enfermagem, Direito, Educação Física, Administração, Ciências Biológicas, Biomedicina, Agronomia, Engenharia Civil, Fisioterapia, Pedagogia e Zootecnia), que concordaram em participar do estudo e assinaram o Termo de Consentimento Livre e Esclarecido (TCLE). Foram excluídos deste estudo os acadêmicos menores de 18 anos que responderem no questionário que não tiveram relações sexuais, que não preencherem o questionário por completo e os que não concordaram em assinar o (TCLE).

A pesquisa foi realizada no Instituto Educacional Santa Catarina - Faculdade Guaraí, FAG/IESC, na região central do Estado do Tocantins. $O$ instituto é uma Instituição privada com localização da sua sede em Santa Catarina, totaliza o total de 11 cursos na cidade de Guaraí/TO, com mais de 1.000 alunos.

A coleta de dados foi realizada no mês de outubro de 2020, através de um questionário online, preenchido pelos pesquisadores.

\section{RESULTADOS E DISCUSSÃO}

Em meio ao século XIX o termo sexualidade surgiu como algo distinto apenas uma mudança no vocabulário, foi a partir daí que a definição desta palavra passou a ser associada com outros acontecimentos, sendo alguns relacionados como: introdução a várias regras e preceitos impostos por instituições religiosas, jurídicas, médicas e pedagógicas; ao avanço, ou evolução, de diversos ramos de conhecimentos; às alterações na forma como os indivíduos são orientados a explicar e dar valor à sua atuação, seus prazeres, desejos, sensações, sentimentos, vontades e sonhos (FIGUEIROA et al., 2017).

No contexto das faculdades e academias, essa discussão se torna ainda mais preocupante e merece toda a atenção, pois mesmo sendo um espaço da construção e da propagação do conhecimento, ainda se tem grandes índices de Infecções Sexualmente Transmissíveis, além da educação sexual e reprodutiva. Sendo assim é preciso entender a problemática e como ela se consolida de maneira tão enfática em um ambiente que deveria ser protegido em função do conhecimento e do fácil acesso a informação (OLLIVEIRA et al., 2016). 
Para o presente estudo tivemos a participação de 146 acadêmicos, a amostra apresentou maior interação dos cursos da área da saúde, com 65 participantes, seguido pelo curso da área das humanas, composta por 58 participantes e pelo curso de engenharias com 23 acadêmicos ativos.

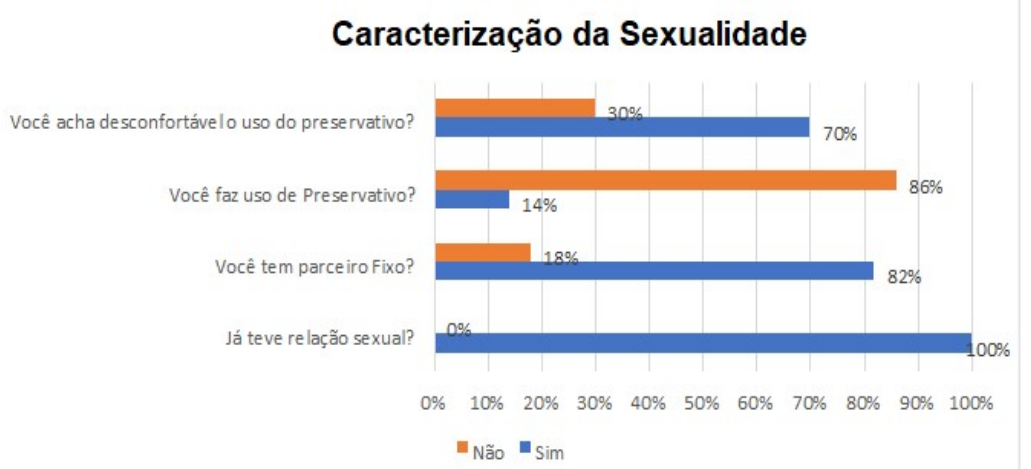

Gráfico 01: Caracterização da sexualidade dos acadêmicos de uma Faculdade no interior do Estado do Tocantins.

Os dados demonstrados no gráfico 01 descrevem à caracterização da sexualidade dos acadêmicos de uma faculdade no interior do estado do Tocantins. Dos dados que são representados no gráfico, 100\% dos entrevistados relataram que já tiveram relação sexual, apontando uma grande relevância dos acadêmicos que são ativos sexualmente.

Dos entrevistados, $82 \%$ possuem parceiro fixo, enquanto $18 \%$ relatam não possuir parceiro efetivo. Nos resultados emergidos sobre o parceiro fixo, a pesquisa foi na contra mão do autor Pinto, pois o mesmo fundamenta que: Existem várias constatações que apontam que entre universitários os maiores problemas estão relacionados com a quantidade de parceiros e consequentemente ao risco de exposição a agentes patógenos e também aos métodos de contracepção que na maioria das vezes são burlados por esses acadêmicos que visam o prazer momentâneo e desprezam a responsabilidade futura, sendo assim, é um problema de enfermagem iminente (PINTO, 2015).

No que se refere ao uso do preservativo, $86 \%$ dos participantes disseram não fazerem seu uso, enquanto $14 \%$ relatou usá-lo com frequência. Dos 146 participantes $70 \%$ relatam que não fazem uso por achar desconfortável, e 30\% não sentem qualquer incômodo.

Segundo a pesquisa realizada, é possível observar o número significativo de acadêmicos que não fazem uso do preservativo. Podemos considerar tal resultado preocupante e ligar ao fato de que esta grande maioria sente certa segurança por terem seus parceiros fixos e vê o uso do preservativo apenas como método contraceptivo e não como um método que evita Infecções Sexualmente Transmissíveis (IST).

Os resultados são concordantes com outros estudos, onde se considera que o uso do preservativo é determinado de acordo com o tipo do relacionamento, fazendo com que seja apropriada a confiança depositada no parceiro. Atestada tal confiança, ambos os parceiros veem como oportuno o abandono do uso do preservativo, supondo que os riscos foram reduzidos pelo fato do compromisso fixo.

Dessa forma, alguns autores supõem que a confiança no parceiro seja um indicador de risco, pois se acredita que os parceiros seriam incapazes de enfrentar uma traição, o que faria da contaminação por IST's uma condição distante. Além do mais, inúmeros estudos indicam ainda que essa confiança depositada nos 
parceiros interfere diretamente no uso do preservativo em relações mais estáveis, pela falta de conhecimento que possuem em relação aos prejuízos que podem causar uma relação desprotegida (PLUTARCO et al., 2019).

Questionados sobre qual o método de anticoncepção utilizado atualmente, 62 participantes relataram fazer o uso do preservativo masculino e feminino (Gráfico 02), nos levando a comparar com o resultado negativo do Gráfico 01 , onde foram questionados sobre o uso do preservativo nas relações.

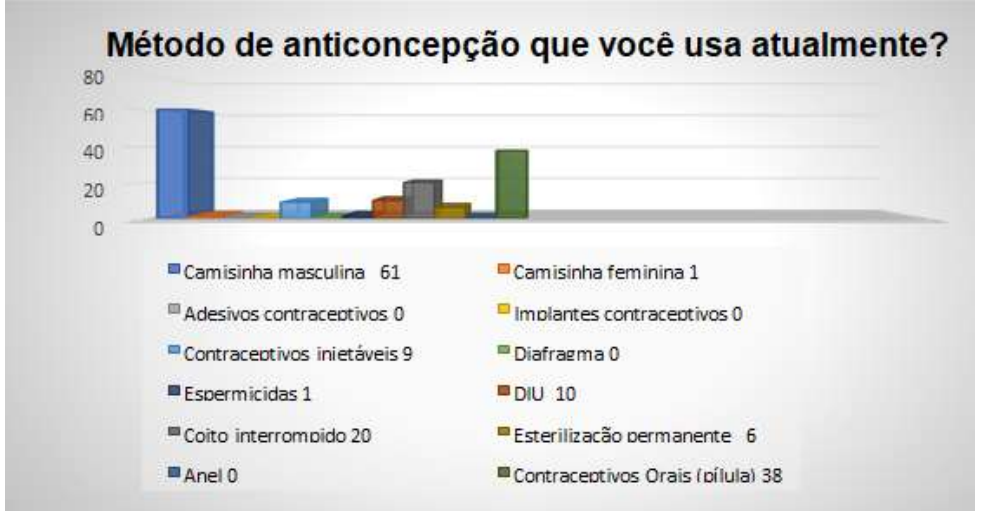

Gráfico 02: Métodos anticoncepcionais utilizados.

Na apreciação do Gráfico 02, é possível observar que de 146 participantes, 62 indivíduos fazem o uso do preservativo masculino e feminino, enquanto um número expressivo de 84 dos entrevistados utiliza os demais métodos contraceptivos, que são: contraceptivos injetáveis (9), contraceptivos orais (38), Espermicidas (1), DIU (10), Coito interrompido (20) e Esterilização permanente (6); deixando claro que a intenção é apenas evitar uma possível gravidez indesejada.

Questionados sobre qual o método de anticoncepção utilizado atualmente, a maioria dos participantes relataram fazer o uso do preservativo masculino e feminino (Gráfico 02), nos levando a comparar com o resultado negativo do Gráfico 01 , onde foram questionados sobre o uso do preservativo nas relações sexuais, no qual $86 \%$ relataram não fazer uso.

Assim como o planejamento familiar, a contracepção é um direito que assiste os casais, pela Constituição Federal através da Lei no 9.263 de 12 de janeiro de 1996, onde assegura que as pessoas tenham liberdade na escolha de querer ou não ter filhos, a quantidade que pretendem ter e o momento que acharem oportuno, bem como o direito as informações, métodos, meios e técnicas para ter ou evitar uma gravidez indesejada (COSTA et al., 2017). Essa atitude confirma os resultados obtidos na pergunta relacionada a educação sexual dos participantes, conforme é possível observar no Gráfico 03.

$\mathrm{Na}$ análise é possível observar que metade dos participantes $(50 \%)$ relataram de maneira negativa sobre a educação sexual recebida, onde $32 \%$ não receberam nenhuma instrução e $18 \%$ tiveram pouco ou quase nenhuma educação sexual.

Com menor expressão, $29 \%$ dos entrevistados responderam de maneira positiva quando questionados quanto à educação sexual recebida, dessa porcentagem obtida $18 \%$ disseram ter adquirido tal educação, de maneira satisfatória, ainda dentro de casa, enquanto $11 \%$ teriam recebido instruções sobre o assunto na escola. 


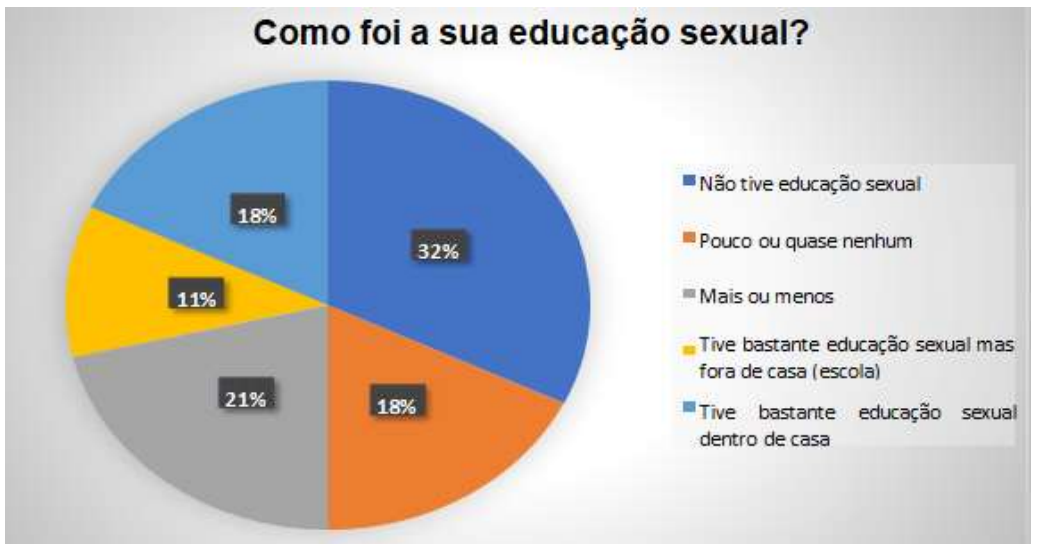

Gráfico 03: Dados sobre a educação sexual dos participantes

A saúde sexual não tem um objetivo limitado a dar conselhos e prestar assistência sobre reprodução e IST's, mas também em relação à melhoria da qualidade de vida e bom desenvolvimento das relações pessoais, ou seja, atua na preparação dos jovens para a fase adulta, possibilitando as reflexões sobre determinadas circunstâncias enquadradas em seu contexto (KERNTOPF et al., 2018).

A educação sexual é um recurso que acontece de maneira extensa e pode ser informal e assistemático ou, ainda, formal e intencional. Sendo assim, precisa ser fundamentada no contexto econômico, social, religioso e cultural de cada jovem, tendo como base a promoção à saúde e a prevenção de IST's e gravidez não desejada (KERNTOPF et al., 2018).

É necessário entender que as práticas sexuais são a resposta final da relação entre fatores pessoais, biológicos, institucionais e sociais. Lamentavelmente poucos estudos, até então, prestaram uma atenção minuciosa voltada apenas para a qualidade sexual dos jovens, buscam sempre focar na prevenção de IST's e gravidez não planejada, deixando passar em branco a importância de se estudar sobre o grau de satisfação sexual em meio a essa população (SERRANO et al., 2013).

Satisfação sexual é um termo que remete diversos significados para as pessoas, e só é definido com base em suas experiências vividas e a forma como atribuem individualmente. De teor multifatorial e biopsicossocial, a satisfação sexual depende exclusivamente do comportamento próprio do casal, isso acontece em decorrência de fatores psicológicos do prazer sexual, que são resultados da cultura e do meio social onde a pessoa se desenvolveu (SERRANO et al., 2013). Os participantes desta pesquisa foram questionados sobre o grau de satisfação sexual em seus relacionamentos, conforme resultados indicados no gráfico 04 .

\section{Qual é o seu grau de satisfação sexual?}

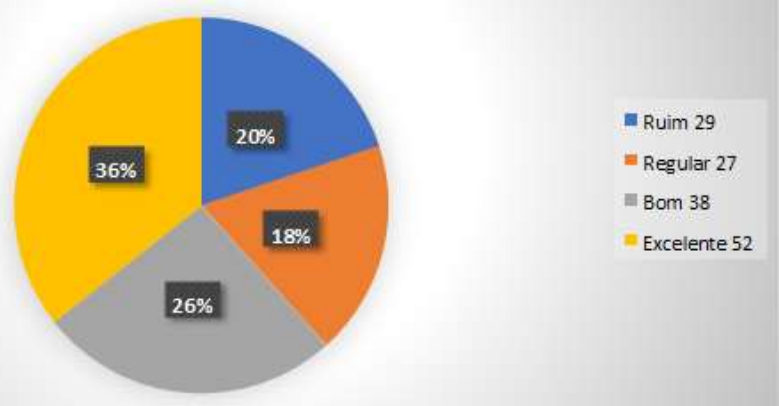

Gráfico 04: Grau de satisfação sexual entre os acadêmicos. 
O resultado mostrou-se positivo (excelente ou bom) em 62\% (90) dos jovens e negativo (ruim) em apenas $20 \%$ (29) dos participantes. A porcentagem restante 18\% (27) aborda um grau de satisfação sexual regular, não se encaixando em nenhuma das estatísticas citadas.

A ideia de satisfação sexual está intimamente relacionada com as práticas sexuais vivenciadas pelo indivíduo, perspectivas atuais e ambições futuras. Já a insatisfação sexual pode ser ocasionada por disfunções sexuais na própria pessoa, em seu parceiro (a), ou também pode existir independente de qualquer disfunção (SILVA et al., 2016).

A sexualidade humana permaneceu sendo um assunto estudado e comentado esporadicamente até meados da década de 60 , do século XX, quando passou a ser admitida como assunto de grande pertinência, tornando-se atualmente um importante objeto de estudo entre os projetos científicos brasileiros (FIGUEIROA et al., 2017). É imprescindível destacar que o estudo da sexualidade tem particularidades complexas e subjetivas, precisando de um olhar holístico e minucioso para que seja entendido adequadamente, por se tratar de um processo constante, que percorre toda a vida, obtendo contínuas ações de diversos fatores (MIRANDA et al., 2015).

A sexualidade é tida ainda como uma das principais características que nos identifica, de maneira universal, como espécie humana, sendo o que há de mais íntimo nos indivíduos. É também uma temática de interesse público, pois o comportamento sexual está ligado diretamente à saúde pública, à natalidade, à propagação da espécie, o que por sua vez, está relacionado ao possível aumento da produção de bens, povoamento e consequente força da sociedade (PEREIRA et al., 2015).

\section{Comportamento sexual entre acadêmicos}

Entre 15 e 30 anos de idade, os jovens adultos ou adultos jovens, experimentam a mudança de uma das fases da vida, marcada pelo fim da adolescência e início da vida adulta, com inúmeras modificações psicológicas e biológicas. Geralmente essa fase coincide com a entrada em uma universidade, e com o início das descobertas que envolvem a sexualidade (ARRIAGADA et al., 2014).

Os jovens adultos, envoltos entre os universitários, compõem um grupo vulnerabilidade e de fácil alvo para os danos alusivos ao comportamento sexual de risco entre os acadêmicos, como a baixa aceitação ao uso de preservativo, trocas frequentes de parceiro, uso corriqueiro de álcool e outras substâncias psicoativas no momento do ato sexual, a fim de afetar a percepção da instabilidade pessoal (FREITAS et al., 2019).

Relacionada a essas características que marcam essa transição de fases na vida humana, o ingresso no Ensino Superior desperta uma série de mudanças e adaptações na vida desses jovens, como consequência dos novos relacionamentos sociais que passam a vivenciar, o que acaba tornando este momento em um período indefeso, propenso a exposição a fatores de risco à saúde, destacando principalmente aqueles relacionados ao estilo de vida, como a prática do sexo desprotegido e o uso de substâncias tóxicas (FIRMEZA et al., 2016).

Em relação à prática da sexualidade, é possível perceber que muitas vezes os jovens acabam 
recebendo informações de procedências incertas. Atualmente vivemos em uma comunidade erotizada, onde os jovens possuem mensagens indefinidas em relação ao bom e o ruim da sexualidade. Existe uma flexibilidade social desleixada, sendo habitual que a atividade sexual seja iniciada sem entendimento satisfatório entre o que se espera e a intervenção recebida pelos parceiros e pela sociedade. É reconhecido que a responsabilidade da educação sexual seja dos pais, no entanto essa realidade acaba não existindo na maioria das famílias, por dificuldade dos próprios pais em lidar com assuntos relacionados a sexualidade dos seus filhos (NASCIMENTO et al., 2018).

Tal ingresso no Ensino Superior evidencia, cada vez mais, a relevância de o acadêmico ser atendido de forma holística, devendo ser compreendido de maneira individual. Sendo assim, torna-se imprescindível que uma Assistência de Enfermagem seja planejada de acordo com a necessidade de cada um, incluindo principalmente o direcionamento na esfera da sexualidade (SANTOS et al., 2007).

A população jovem é o grupo populacional mais vulnerável às Infeç̧ões Sexualmente Transmissíveis (IST) nos aspectos biopsicosocioculturais e programáticos. No que diz respeito a educação em saúde sobre as IST, estudo revelam que os universitários conhecem o mínimo possível sobres essas infecções e grande parte desconhece os principais sintomas, formas de propagação e prevenção, principalmente quando se trata de infecções de grande incidência, porém com abordagem baixa nos diversos meios de informação, como é o caso da tricomoníase e clamídia (FONTE et al., 2018). Além do mais, ainda existem muitos outros fatores de risco que precisam de uma atenção especial, entre eles podemos citar a gravidez indesejada, que acomete grande parte da população jovem.

\section{Principais fatores de risco, relacionados à falta de informação sobre sexualidade}

A educação sexual baseia-se em disponibilizar condições para que as pessoas aceitem a sua sexualidade e o seu corpo com posicionamento positivo, desprendido de preconceitos, medos, vergonhas, culpas, tabus e bloqueios. Hoje em dia, em nossa sociedade, a sexualidade vem sendo vista e/ou abordada de maneira que as pessoas sejam doutrinadas a conhecê-la, aprendendo que tal ato não é algo feio e condenável (GONÇALVES et al., 2013).

A falta de diálogo sobre a temática "sexualidade" facilita a exposição de jovens e adultos a situações de riscos referentes ao exercício da sexualidade, como o contágio por Infecções Sexualmente Transmissíveis (IST), gravidez indesejada, traumas psicológicos e emocionais decorrentes de experiências sexuais frustrantes (GONÇALVES et al., 2013).

As Infecções Sexualmente Transmissíveis (IST) são de caráter contagioso, sendo produzidas por mais de trinta fontes etiológicas (bactérias, vírus, protozoários e fungos), transmitidas por contato sexual direto ou indireto e eventualmente por via sanguínea, que acontece quando há o compartilhamento de seringas para o uso de drogas; durante a gestação, da mãe para o bebê; entre outras (PASSOS et al., 2017).

Em relação a gravidez indesejada, ou não planejada, existem diferentes causas que podem explicar o aumento da sua taxa em meio aos jovens, o que pode variar entre os países. Um dos fatores de risco que podemos destacar nesta complexidade, são os aspectos socioeconômicos. Mesmo que esse fenômeno 
atinja e cresça continuamente em todas as classes sociais, ainda é evidente que existe uma forte relação entre a pobreza e a baixa idade ao engravidar (CERQUEIRA et al., 2010).

Contudo, uma gravidez não planejada indica, sistematicamente, a exposição direta a, pelo menos, uma circunstância que causam algum prejuízo, sendo o sexo desprotegido o grande vilão (CERQUEIRA et al., 2010).

Falando-se em direitos reprodutivos, é evidente que a liberdade de escolha acometa a área da regulação da fecundidade. No entanto, para que as pessoas, em especial as mulheres, possam optar por um método contraceptivo de maneira livre e informada, é necessário que conheçam e tenham acesso a todos os cientificamente aprovados e disponíveis, escolhendo aquele que seja mais apropriado às suas condições de vida ou vontade própria (ALMEIDA, 2010).

\section{CONCLUSÕES}

O comportamento sexual dos acadêmicos entrevistados a partir do conjunto analisado, observa-se que, para o fato, a sexualidade na formação acadêmica é tratada a partir de uma atitude de casualidade e informalidade, surgindo associada à educação de algumas técnicas isoladas, em algumas atividades complementares da graduação e nos grupos de investigação.

Desse modo, reforça-se que a sexualidade precisa ser tratada como assunto de estudo na academia como fenômeno essencial a todo o ser humano. Nesse sentido, acredita-se ser necessário gerar espaços de discussão e reflexão que tratem não apenas da sexualidade do sujeito cuidado, mas, também, dos estudantes pesquisados.

A abrangência da sexualidade pelos docentes permite a eles não apenas romper com a cultura do sigilo e desvelar essa temática na formação acadêmica, mas, também, tratar essa questão para além dos limites da apreensão, rompendo com tabus e preconceitos.

De maneira geral, as argumentações sobre sexualidade devem ser vias de regras em diferentes ambientes sociais. Preferencialmente esta discussão sobre sexualidade precisa ser desenvolvida antes da entrada na vida acadêmica, ainda no início da adolescência, levando em consideração uma possível antecipação do início das práticas sexuais. Porém, as faculdades também podem contribuir positivamente na formação sexual desses jovens, principalmente entre acadêmicos da área da saúde, que dependerão desses conhecimentos na atuação profissional.

Conclui-se então que os resultados indicam falhas no auto cuidado dos jovens, que adotaram comportamentos errôneos pela ausência de educação sexual. Nesse caso é de extrema importância que reflexões sejam discutidas e promovidas em relação aos comportamentos sexuais que oferecem risco e a fragilidade em que esses jovens são expostos diariamente, mesmo com parceiro (a) fixo, fazendo com que sejam conscientizados sobre os problemas e assumam uma postura comprometida com a prevenção da saúde.

Ademais, não se teve a ambição, com esta busca, de extenuar a temática em estudo, sendo estimada como formidável a percepção de novos olhares sobre a sexualidade. Espera que foi possível 
colaborar para ponderações especiais acerca da sexualidade na formação acadêmica do IESC, o que se viabilizou tanto a partir daquilo que foi dito pelos estudantes quão do que tenha ficado por eles cultivado escondido.

\section{REFERÊNCIAS}

ALMEIDA, L. C.. Métodos Contraceptivos: uma revisão bibliográfica. Contagem: Universidade Federal de Minas Gerais, 2010.

ARRIAGADA, E. M. B.; BERTONI, J. S.. Una revisión de los estilos de vida de estudiantes universitários Iberoamericanos. Cienc Enferm, v.20, n.2, p.93-101, 2014

CARNEIRO, R. F.; SILVA, N. C.; ALVES, T. A.; ALBUQUERQUE, D. O.; BRITO, C. D.; OLIVEIRA, L. L. Educação sexual na adolescência: uma abordagem no contexto escolar. Sanare, v.14, n.1, p.104-108, 2015.

CERQUEIRA, S.. Gravidez na adolescência: risco e proteção. Psicologia em Estudo, v.15, n.1, p.73-85.

COSTA, A. G. S.. Práticas contraceptivas entre universitárias da faculdade de medicina de Valença - RJ. Brazilian Journal of Surgery and Clinical Research, v.19, n.1, p.64-70, 2017.

FIGUEIROA, M. N.. A formação relacionada com a sexualidade humana na percepção de estudantes de enfermagem. Rev. Enf. Ref., v.4, n.15, p.21-30, 2017.

FIRMEZA, S. N. R. M.. Comportamento sexual entre acadêmicos de uma universidade pública. Rev Rene, v.17 n.4, p.506-11.

FONTE, V. R. F.; SPINDOLA, T.; FRANCISCO, M. T. R.; SODRÉ, C. P.; ANDRÉ, N. L. N. O.; PINHEIRO, C. D. P.. Conhecimento das infecções sexualmente transmissíveis entre universitários. Esc Anna Nery, v.22, n.2, 2018.

FREITAS, J. L. G.; PEREIRA, P. P. S.; MOREIRA, K. F. A.; SILVA, A. D.. Prevalência do não uso de preservativo entre universitários e pós-graduandos de uma universidade pública do Norte do Brasil. Revista Eletrônica Acervo Saúde, v.25, 2019.

GONÇALVES, R. C.; FALEIRO, J. H.; MALAFAIA, G.. Educação sexual no contexto familiar e escolar: impasses e desafios. HOLOS, v.5, 2013.

KERNTOPF, R. M.. Sexualidade na adolescência: uma revisão crítica da literatura, Adolescência e Saúde, 2018.
MIRANDA, C. S.; SANTOS, V. N.; OLIVEIRA, G. F.. A sexualidade sob o olhar transdisciplinar: desafios para a prática docente. In: CONGRESSO NACIONAL EM EDUCAÇÃO, 12. Anais. Curitiba: PUCPR. 2015.

NASCIMENTO, B. S.. Comportamento sexual de jovens universitários e o cuidado com a saúde sexual e reprodutiva. Enferm. Glob., v.17, n.49, p.237-269, 2018.

OLIVEIRA, H. F.. Guia prático de atualização departamento científico de adolescência prevenção da gravidez na adolescência. 2016.

PASSOS, T. S.; HORA, A. B.; PAIXÃO, A. L. S. S.. Educação em Saúde para Prevenção de Infecções Sexualmente Transmissíveis em Comunidades Quilombolas. Rev Enferm UFPE, v.11, n.10, p.3965-70, 2017

PEREIRA, Z. M.; MONTEIRO, S.. Gênero e sexualidade no ensino de ciências no Brasil: análise da produção científica. Revista Contexto e Educação, v.30, 2015.

PINTO, A. F.. Sexualidade na adolescência. 2015

PLUTARCO, L. W.. A influência da confiança no parceiro na decisão do uso da camisinha. Psic., Saúde \& Doenças, v.20, n.1, p.220-233, 2019.

SANTOS, L. V.. Sexualidade Humana: conhecimentos dos Acadêmicos de Enfermagem. Esc Anna Nery R Enferm, v.11 n.2, p.303-6, 2007.

SERRANO, A. G.. ZABALGOITIA, M. T. H. M.. Práticas e satisfação sexual em jovens universitários. Revista Latinoamericana de Medicina Sexual, v.2, n.2, 2013.

SILVA, P. O.; LILA, L. C.; VALLE, P. A. S. S.. Interferência no desenvolvimento da sexualidade de mulheres universitárias da área de saúde. Revista Brasileira de Sexualidade Humana, v.27, n.2, p.17-24. DOI: https://doi.org/10.35919/rbsh.v27i2.107. RBSH 2016

A CBPC - Companhia Brasileira de Produção Científica (CNPJ: 11.221.422/0001-03) detém os direitos materiais desta publicação. Os direitos referem-se à publicação do trabalho em qualquer parte do mundo, incluindo os direitos às renovações, expansões e disseminações da contribuição, bem como outros direitos subsidiários. Todos os trabalhos publicados eletronicamente poderão posteriormente ser publicados em coletâneas impressas sob coordenação da Sustenere Publishing, da Companhia Brasileira de Produção Científica e seus parceiros autorizados. Os (as) autores (as) preservam os direitos autorais, mas não têm permissão para a publicação da contribuição em outro meio, impresso ou digital, em português ou em tradução. 